\title{
Tratamiento farmacológico del tabaquismo
}

\author{
SERGIO BELLO S.*, ALVARO FLORES C.** y MAGDALENA BELLO S.**
}

\section{Pharmacotherapy for tobbaco dependence}

Currently, tobacco smoking is considered to be a chronic systemic disease that belongs to the group of addictions. Nicotine is the responsible substance of this dependence, producing a variety of disorders at the central nervous system, mainly through a raise of the dopamine levels. Tobacco dependence treatment has two main branches: psychosocial and pharmacological. Nicotine replacement therapy, Bupropion and Varenicline have been approved by the FDA as first-line treatments for nicotine dependence. There are some second-line drugs, such as Clonidine and Nortriptyline that have shown less efficacy and more adverse effects. Currently there are many other drugs in research, such as antinicotine vaccine and Rimonobant, that will probably be available for its use in the coming years. The current review analyzes the pharmacological choices existing today for treating tobacco dependence and those coming in the future.

Key words: Smoking, nicotine, drug therapy, tobacco use disorder.

\section{Resumen}

Actualmente se considera que el tabaquismo es una enfermedad crónica sistémica, perteneciente al grupo de las adicciones. La nicotina es la sustancia responsable de esta dependencia, produciendo una serie de alteraciones en el SNC, principalmente mediante el aumento de dopamina. El tratamiento del tabaquismo incluye dos componentes: psicosocial y farmacológico. Hasta la actualidad, la FDA ha aprobado 3 medicamentos que han demostrado ser eficaces: Terapia de reemplazo de Nicotina, Bupropión y Vareniclina. Además existen drogas de segunda línea, como la Clonidina y Nortriptilina, menos eficaces y con mayores efectos adversos. Actualmente, hay muchos otros medicamentos en investigación, entre otros la Vacuna antinicotina y el Rimonobant, que probablemente estarán disponibles para su uso en los próximos años. En este artículo se analizan las alternativas farmacológicas existentes hoy en día para el tratamiento del tabaquismo y lo que podría venir en el futuro.

Palabras clave: Tabaquismo, adicción, nicotina, farmacoterapia.

\section{Introducción}

El consumo de tabaco en Chile se ha convertido en uno de los factores de riesgo más importantes de enfermedad y muerte, estimándose como factor causal de 15.000 muertes anuales, que constituyen el $17 \%$ de todas las muertes ${ }^{1}$. Estas cifras están condicionadas porque en el país existe una alta prevalencia de consumo, tanto en adultos ${ }^{2-4}$ como en jóvenes ${ }^{5}$ teniendo el triste récord de ser el país de las Américas con el más alto consumo entre los jóvenes ${ }^{6}$.
Es por ello que es considerado como una prioridad para la salud pública nacional el control de su consumo. El Ministerio de Salud ha planteado la necesidad de la disminución de prevalencias dentro de los objetivos sanitarios ${ }^{7}$ para el año 2010, tanto en adultos como en jóvenes.

El tabaquismo es la principal causa de diversas enfermedades respiratorias y uno de los principales factores de riesgo de enfermedad cardiovascular, cáncer y de muchas otras patologías ${ }^{8}$. En el año 2000, las muertes relacionadas con el

\footnotetext{
* Instituto Nacional del Tórax.

** Internos de Medicina. Pontificia Universidad Católica de Chile.
} 
tabaquismo en el mundo fueron alrededor de 4,8 millones, cuyas principales causas fueron en orden de frecuencia: enfermedades cardiovasculares, EPOC y cáncer pulmonar'. Se ha visto que los fumadores mueren en promedio 10 años antes que los no fumadores y que la cesación a los 60, 50, 40 ó 30 años aumenta la expectativa de vida en 3, 6, 9 ó 10 años respectivamente ${ }^{10}$.

Este artículo tiene una primera parte sobre las bases de los mecanismos de adicción al tabaco y luego, una segunda parte con enfoque clínico, que incluye aspectos generales del tratamiento y una revisión de las terapias farmacológicas disponibles hoy en día.

\section{Tabaquismo, enfermedad adictiva crónica}

Desde 1988, año en que se publicó el Informe del Cirujano General titulado "Adicción a la Nicotina" 11 existe sólida evidencia de la condición adictiva del consumo de tabaco. Las principales conclusiones de ese informe fueron que el tabaco es adictivo, que la nicotina es la droga que causa la adicción y que el proceso psicofarmacológico involucrado en esta adicción es similar al de otras drogas, tales como la heroína o la cocaína.

En la actualidad se considera que el tabaquismo es una enfermedad crónica sistémica que pertenece al grupo de las adicciones, clasificada

\section{Tabla 1. Criterios DSM-IV de dependencia} a la nicotina

1. Tolerancia: disminución del efecto con una cantidad constante de la sustancia, con necesidad de cantidades crecientes de la sustancia para conseguir el efecto deseado

2. Abstinencia: aparición de un síndrome de abstinencia característico ante la falta de la sustancia

3. Fumar cantidades mayores o por un período más largo que el que se desea

4. Deseo persistente de fumar e intentos fallidos para disminuir o interrumpir el consumo

5. Utilizar considerables períodos de tiempo en actividades relacionadas con la obtención o el consumo del tabaco

6. Reducción de actividades sociales, laborales o recreativas por fumar

7. Continuar fumando a pesar de tener conciencia del daño que ocasiona a la salud dentro del Manual diagnóstico y estadístico de los trastornos mentales (DSM IV) de la American Psychiatric Association ${ }^{12}$. Según esta clasificación, la dependencia a la nicotina se diagnostica por la presencia de 3 ó más de los siete criterios propuestos (Tabla 1), durante un período continuo de 12 meses, produciendo un deterioro o malestar clínicamente significativo. A su vez se específican los criterios para el diagnóstico de la abstinencia de nicotina (Tabla 2). La Clasificación Internacional de Enfermedades (CIE-10) de la Organización Mundial de la Salud también incluye el tabaquismo dentro de los "Trastornos mentales y del comportamiento debidos al consumo del tabaco", en la sección F17, cuyos criterios son muy similares a los del DMS IV (en vez de 7 criterios se condensan en 5 y se agrega un sexto en relación al deseo intenso por consumir la sustancia) $)^{13}$.

El origen de la adicción es multifactorial, implicando factores biológicos, genéticos, psicológicos y sociales. Las manifestaciones clínicas dependen de las características individuales de personalidad de cada individuo, así como de las circunstancias socio-culturales que lo rodean. El principal síntoma es la necesidad imperiosa o compulsiva de volver a consumir la sustancia para experimentar la recompensa que produce y para evitar el síndrome de abstinencia.

Si bien, el tabaco contiene miles de sustancias, es la nicotina la que más frecuentemente se asocia con la dependencia. La nicotina es una

Tabla 2. Criterios DSM-IV del Síndrome de abstinencia de nicotina

1. Consumo de nicotina durante al menos algunas semanas

2. Interrupción brusca o disminución de la cantidad de nicotina consumida, con al menos 4 de los siguientes síntomas en las 24 horas siguientes:
a. Ánimo disfórico o depresivo
b. Insomnio
c. Irritabilidad, frustración, ira
d. Ansiedad
e. Dificultades de concentración
f. Inquietud
g. Disminución de la frecuencia cardíaca
h. Aumento del apetito o del peso

3. Los síntomas provocan un malestar clínicamente significativo o un deterioro social o laboral del individuo

4. Los síntomas no se deben a una enfermedad médica ni se explican mejor por la presencia de otro trastorno mental 
droga psicoactiva generadora de dependencia, con conductas y alteraciones físicas específicas, que conduce a que el adicto fume, a pesar de conocer o experimentar las consecuencias negativas para su salud.

Estructuralmente, la nicotina es una amina terciaria. Su absorción es principalmente a nivel alveolar, siendo ésta muy rápida, por la gran superficie de contacto de los alvéolos y la disolución de la nicotina en fluidos con $\mathrm{pH}$ fisiológico. En forma minoritaria se absorbe en la mucosa bucal, donde es dependiente del $\mathrm{pH}$, ya que el humo de los cigarrillos es ácido ( $\mathrm{pH} 5,5)$ y por lo tanto, la nicotina se encuentra ionizada, dificultando su absorción.

Después de inhalar el humo, en sólo 9 segundos, la nicotina llega hasta el cerebro. Luego, sus niveles cerebrales declinan rápidamente ya que se distribuye en otros tejidos (ganglios autonómicos, médula suprarrenal y uniones neuromusculares) y se metaboliza en el hígado, por enzimas de la familia CYP2A6. Finalmente sus metabolitos se excretan a través del riñón, siendo su vida media de $2 \mathrm{~h}$ aproximadamente ${ }^{14}$.

La nicotina actúa a través de su unión a los receptores nicotínicos de acetilcolina (nAch), cuya estructura corresponde a canales iónicos compuestos por 5 subunidades. Existen alrededor de 17 subunidades diferentes y la combinación de éstas le confiere a cada receptor distintas propiedades. Se ha demostrado que en la adicción a la nicotina los receptores $\alpha 4 \beta 2$ tienen un papel determinante por su alta afinidad y sensibilidad a la nicotina. Están formados por dos subunidades $\alpha 4$ y tres $\beta 2$ y se ubican principalmente en el área tegmental ventral (dentro del circuito de recompensa del cerebro). La subunidad $\alpha 4$ tendría mayor importancia en la producción de la sensación de placer al fumar y la subunidad $\beta 2$ en la conducta de autoadministración asociada a la nicotina ${ }^{15}$.

Los receptores nicotínicos pueden estar en 3 estados: 1) reposo: canal iónico cerrado, que puede abrirse con la unión con el agonista (Ach o nicotina); 2) activado: se une el agonista y el canal se abre, produciéndose un flujo de iones de $\mathrm{Na}+$ hacia el interior, induciendo una depolarización local; 3) desensibilizado: después de la activación, se cierra el canal iónico y se hace refractario a la activación (se necesitarían altas concentraciones del agonista para activarlo) ${ }^{15}$.

La nicotina produce una serie de alteraciones en el SNC que explicarían su poder adictivo. Estos efectos son:

1. Aumento de dopamina en el núcleo accumbens: en el SNC la nicotina se une princi- palmente a receptores del área tegmental ventral, que tiene proyecciones hacia el núcleo accumbens, donde se libera dopamina en el espacio extra-sináptico. Esto constituye el sistema dopaminérgico mesolímbico, que tiene aferencias hacia la corteza frontal, estriada e hipocampo, y que corresponde al circuito de recompensareforzamiento del cerebro. Por la liberación de la dopamina en el núcleo accumbens se produce la sensación de placer y bienestar, lo que determina la dependencia física ${ }^{14}$.

2. Incremento del número de receptores nicotínicos funcionales ("up-regulation"): ocurre por la exposición crónica a nicotina, lo que conlleva a un aumento importante de la concentración extrasináptica de dopamina, produciendo sensaciones más placenteras, lo que está relacionado con el reforzamiento y la adicción a nicotina. Se cree que este incremento de los receptores ocurre como respuesta adaptativa a la desensibilización permanente de éstos, contrario a lo que ocurre con otras drogas adictivas, en que hay una baja de los receptores. Es posible que la tolerancia a la nicotina se explique por la persistencia de los receptores en estado de desensibilización ${ }^{14}$.

3. Sensibilización del sistema mesolímbico: por la exposición repetida a nicotina, este sistema desencadena una respuesta dopaminérgica más fácilmente, lo cual también está relacionado con el incremento del número de receptores. Con el tiempo, el ritual previo al fumar (abrir la cajetilla, sacar el cigarrillo, tenerlo en los dedos) es capaz de estimular la producción de dopamina en el sistema mesolímbico, incluso sin la presencia del agonista, lo que actúa como un elemento reforzador ${ }^{15}$.

4. Disminución de los niveles de monoaminooxidasa B (MAO-B): la nicotina u otra sustancia del humo del tabaco reduce los niveles de MAO-B, que es la enzima encargada de catabolizar la dopamina en el SNC y otros sitios. Esto contribuiría a incrementar aún más la dopamina en el SNC ${ }^{14}$.

Son muchos los factores psicofarmacológicos que contribuyen a la adicción a la nicotina. $\mathrm{Si}$ bien, el sistema dopaminérgico es el que más se ha estudiado por su importancia en la recompensa, es necesario mencionar que la nicotina también actúa en otros sistemas cerebrales que ayudan a mediar en sus efectos adictivos (colinérgico, gabaérgico, glutamaérgico, serotoninérgico, noradrenérgico, opiáceo y endocannabinoide ${ }^{16}$. Los avances que se logren en el tema, serán de gran utilidad en el futuro para el progreso de la farmacoterapia de esta adicción. 


\section{Tratamiento del tabaquismo}

En la actualidad existe consenso que las intervenciones de cesación del tabaquismo deben tener dos componentes: tratamiento psico-social y tratamiento farmacológico. Ambos deberían incluirse en el apoyo a cualquier persona que solicite ayuda para dejar de fumar.

Las estrategias psico-sociales, que mayoritariamente tienen un enfoque cognitivo-conductual, están destinadas a que el fumador reconozca su adicción, sus características personales, modifique pautas de comportamiento, desarrollando estrategias y habilidades para lograr y mantenerse en abstinencia, lo que habitualmente significa cambiar rutinas y estilos de vida, es decir aprender a "vivir sin tabaco".

Las estrategias psico-sociales constituyen el componente más importante de los tratamientos para dejar de fumar, en cualquiera de sus formas (terapias individuales o grupales, líneas telefónicas, materiales de autoayuda, etc), ya que, como se ha dicho previamente, el tabaquismo es una adicción con aspectos psicológicos y conductuales, además del componente físico de adicción a la nicotina ${ }^{17}$.

A continuación nos referiremos en más profundidad al tratamiento farmacológico, tema en cual ha habido importantes avances en los últimos años.

\section{Farmacoterapia del tabaquismo}

Hasta la actualidad, la FDA (Food and Drug Administration de EE.UU.) ha aprobado el uso de 3 tipos de medicamentos en el tratamiento para dejar de fumar: Terapia de Reemplazo de Nicotina (TRN), Bupropión y Vareniclina. Hay otros dos medicamentos, que aunque no están aprobados por la FDA, se utilizan como drogas de segunda línea, como son Clonidina y Nortriptilina. Por último hay una serie de nuevas drogas, en distintas etapas de investigación y que abren interesantes vías de desarrollo, que se describirán más adelante.

\section{Terapia de Reemplazo de Nicotina (TRN)}

Los medicamentos más estudiados y usados para el manejo de la dependencia al tabaco son aquellos que contienen nicotina. Diversos estudios clínicos han demostrado que son seguros y eficaces, aumentando al doble la tasa de éxito de la terapia conductual.

Parecieran ser 3 sus principales mecanismos de acción: reducción de síntomas de privación, reducción de efectos reforzantes y la producción de ciertos efectos previamente buscados en los cigarrillos (relajación, facilitación del enfrentamiento de situaciones estresantes, etc) ${ }^{18}$. El uso de la TRN debe comenzar el día en que se deja de fumar.

Algunos efectos adversos son comunes a todos los productos de TRN, siendo los más frecuentes mareos, náuseas y cefalea. Las contraindicaciones comunes a toda TRN son enfermedades cardiovasculares graves, arritmias cardíacas severas, hipertensión arterial no controlada y accidente vascular encefálico reciente ${ }^{19}$.

Existen distintas formulaciones de reemplazo de nicotina, que pueden ser usadas en forma aislada o asociadas con otros medicamentos.

\section{Chicle de nicotina}

Corresponde al método de TRN más estudiado y ampliamente usado desde los años $80 \mathrm{~s}^{20}$. Actualmente en EEUU está disponible en diversos sabores (menta, naranja y fruta) y en formulaciones de 2 y $4 \mathrm{mg}$, esta última dosis recomendada para fumadores de más de 25 cigarrillos/día. En Chile, existe sólo la presentación con sabor a nicotina de $2 \mathrm{mg}$,

Si bien los niveles plasmáticos alcanzados son relativamente bajos comparados con el cigarrillo, se ha comprobado como un método efectivo para la dependencia del tabaco ${ }^{21}$.

Los pacientes deben ser instruidos para masticar el chicle hasta que se sienta un leve sabor a pimienta, indicando la liberación de nicotina. En ese momento se debe mantener en la boca sin masticar, para luego de algunos minutos volver a hacerlo. No debiera ser usado junto a bebidas o comidas ácidas, ya que el $\mathrm{pH}$ ácido disminuye la absorción de nicotina en la mucosa oral $^{22}$.

Hay dos formas de indicar chicles: por horario, en que la dosis inicial recomendada es de un chicle cada 1 a 2 horas por 6 semanas, luego una unidad cada 2 a 4 horas por tres semanas, y luego 1 chicle cada 4 a 8 horas por tres semanas. Otra alternativa de uso es ad-libitum, es decir, cuantos chicles sean necesarios de acuerdo a la urgencia por fumar y especialmente en situaciones estimulantes para fumar. Sin embargo, se ha observado que con frecuencia los pacientes utilizan dosis más bajas que las recomendadas, disminuyendo así la efectividad de este método.

\section{Parches de nicotina}

Los parches de nicotina entregan una dosis estable de nicotina por 16 a 24 horas. Requiere 
poco esfuerzo para su uso, lo que facilita la adherencia al tratamiento. Están disponibles en dosis de 7, 14 y $21 \mathrm{mg}$. Se recomienda que aquellos pacientes que fuman más de 10 cigarrillos al día comiencen con la dosis de $21 \mathrm{mg} / 24$ horas, siendo esta dosis titulable según respuesta clínica o con mediciones seriadas de cotinina (realizadas mientras el paciente aún fuma). Se ha recomendado usarlos durante 10 semanas, disminuyendo las dosis en las últimas 4 , aunque muchas veces se necesitan períodos de tratamiento más largos.

El efecto adverso más frecuente es irritación localizada de la piel. El uso del parche durante la noche pareciera tener la ventaja de disminuir la urgencia por fumar matinal, pero podría aumentar el riesgo de tener trastornos del sueño, sobre todo sueños vívidos y pesadillas. En estos pacientes se recomienda retirar el parche al acos$\operatorname{tarse}^{23}$.

\section{Spray nasal de nicotina}

El spray nasal libera nicotina directamente en la mucosa nasal. Dentro de las TRN, es el método que más rápidamente reduce los síntomas de privación ${ }^{24}$. Se usa un puff en cada fosa nasal, liberando en total $1 \mathrm{mg}$ de nicotina.

\section{Inhalador de nicotina}

Se trata de un producto diseñado para satisfacer las necesidades del ritual mano-boca. Consiste en un dispositivo que contiene un cartridge o cartucho de nicotina y un sistema vaporizador que se usa en la boca. Este no es un verdadero inhalador, ya que la nicotina prácticamente no llega hasta los alvéolos.

Estas dos últimas formas de TRN, además del losenge (comprimido que se debe disolver en la boca), no están disponibles en Chile.

\section{Nuevas formas y usos de la TRN}

\subsection{Chicles de liberación rápida}

Método de rápida liberación de nicotina, vía mucosa oral, de reciente aparición. Se diferencia del chicle convencional en lograr un alivio más rápido y completo de los síntomas de privación durante los primeros 3 minutos de uso ${ }^{25}$. Se requieren más estudios de eficacia para su empleo de forma masiva.

\subsection{Formas combinadas de TRN}

Existen básicamente 2 formas de administración de nicotina: pasiva y activa. La forma pasiva corresponde a la liberación sostenida de nicotina durante el día, sin presentar un peak plasmático marcado, como actúan, por ejemplo los parches de nicotina. El resto de los medicamentos de TRN se denominan como formas activas; ya que dependen de la decisión "activa" de la persona para utilizarlos. Tienen vida media corta y producen un peak plasmático de nicotina que simula el efecto del cigarrillo, pero de menor intensidad $^{21}$.

La asociación de un medicamento de entrega pasiva, más otro producto de entrega activa que permita la autoadministración en los momentos de urgencia por fumar, ha permitido potenciar el efecto de cada uno por separado ${ }^{26,27}$. La combinación de productos más usada es la de parche más chicle administrado ad-libitum.

\subsection{Reducción de consumo}

Una estrategia para fumadores no motivados en la actualidad o que se sienten incapaces de la cesación completa, es el uso de TRN como parte de una estrategia de "reducción de consumo". Esto consiste en estimular al fumador a disminuir el consumo a la mitad, utilizando métodos de entrega activa. Si después de 3 meses no ha bajado el consumo, se suspende la TRN. Si éste ha disminuido, se continúa hasta por 1 año, poniendo como meta los 6 meses para abstenerse totalmente ${ }^{28}$.

\section{Bupropion}

El bupropión fue el primer fármaco no nicotínico aprobado para el tratamiento del tabaquismo. Es un antidepresivo monocíclico que actúa inhibiendo la recaptación de noradrenalina (NA) y dopamina (DA) en determinadas áreas del cerebro. Aún no se conoce cómo actúa exactamente para el abandono del tabaquismo, pero se cree que es en parte por su efecto en los niveles de DA y NA ${ }^{29}$. Al disminuir la actividad de las neuronas dopaminérgicas, se desactiva el circuito de recompensa y por lo tanto disminuye el deseo de fumar. Además, al disminuir la actividad de las neuronas noradrenérgicas, se lograrían disminuir los síntomas de abstinencia, ya que se cree que el origen de estos síntomas es el aumento de la actividad de las neuronas liberadoras de NA en el locus coeruleus. Otro mecanismo que contribuiría es que el bupropion podría ser un inhibidor no competitivo de los receptores colinérgicos nicotínicos.

\section{Farmacodinamia:}

Se administra por vía oral y se absorbe en el tubo digestivo, sin que la ingesta de alimentos interfiera, con una biodisponibilidad de al menos un $87 \%$. Se metaboliza en el hígado por el citocromo P450 (isoenzima CYP2B6), dando lu- 
gar a su metabolito activo principal, que es el hidroxibupropion, cuya concentración máxima plasmática se alcanza a las 6 horas de su administración. La eliminación se produce principalmente a través de la orina, con una vida media de 21 horas aproximadamente ${ }^{30}$.

\section{Eficacia terapéutica:}

La eficacia de bupropion para dejar de fumar está claramente demostrada. Un meta-análisis reciente, que incluyó 12 ensayos clínicos randomizados, demostró un odds ratio (OR) de 1,56 (IC95\% 1,1-2,21) a los 12 meses en comparación con placebo ${ }^{31}$.

En relación a la disminución de las recaídas por la prolongación del tratamiento (1 año versus 7 semanas), se ha visto un efecto limitado, ya que retrasa las recaídas pero no mejora las tasas de abstinencia a largo plazo $^{30}$. También se ha demostrado que el bupropion reduce el aumento de peso que ocurre al dejar de fumar y que alivia los síntomas de abstinencia, como el mal humor, ansiedad, dificultad de concentración, tristeza y deseo de fumar ${ }^{32}$.

A pesar de que el bupropion es eficaz para el tratamiento del tabaquismo en fumadores con o sin depresión, se cree que los pacientes con depresión o trastornos de ansiedad se podrían beneficiar más con bupropion que con otro tipo de medidas ${ }^{29}$.

\section{Efectos secundarios:}

La dosis recomendada de $300 \mathrm{mg} /$ día es generalmente bien tolerada. Aproximadamente un $10 \%$ de los pacientes deben suprimirlo por los efectos secundarios ${ }^{33}$.

Los efectos más frecuentes son insomnio (frecuencia de un 30-45\% de los que usan $300 \mathrm{mg} /$ día), sequedad de boca (5-15\%) y náuseas.

Otros efectos secundarios más graves que se pueden observar son las convulsiones y reacciones de hipersensibilidad, cada uno con una incidencia de $0,1 \%$. Las convulsiones probablemente están relacionadas con la dosis y aparecen más frecuentemente en personas que presentan alguna predisposición o en pacientes con insuficiencia hepática o renal. Se han descrito casos de hipersensibilidad retardada y de reacciones anafilácticas.

\section{Recomendaciones de uso:}

Bupropion está recomendado como fármaco de primera línea en el tratamiento del tabaquis$\mathrm{mo}^{32}$. La dosis máxima recomendada es de 150 mg dos veces al día, comenzando una o dos semanas antes de la fecha fijada para dejar de fumar. Los primeros 5-7 días se administra una dosis de $150 \mathrm{mg}$ en la mañana y luego se puede añadir la segunda dosis de $150 \mathrm{mg} 8$ horas después de la primera (para evitar el insomnio). La duración recomendada del tratamiento es 7-9 semanas. Si aparecen efectos secundarios puede disminuirse la dosis a $150 \mathrm{mg} /$ día.

Se debe usar con precaución en pacientes con mayor riesgo de convulsiones: abuso de alcohol o cocaína, o uso de otros fármacos como antipsicóticos, antidepresivos, teofilina, tramadol, quinolonas, corticoides sistémicos o antihistamínicos sedantes. En pacientes adultos mayores y/o con insuficiencia hepática o renal o diabetes, también se debe tener precaución, usando menores dosis que las habituales $(150 \mathrm{mg} /$ día).

Se ha visto que por el uso concomitante de bupropion y parches de nicotina hay un aumento de la incidencia de hipertensión arterial, por lo que se debe tener cuidado con esta asociación en pacientes que tienden al alza de su presión.

Está contraindicado su uso en pacientes con antecedentes de convulsiones, tumor del SNC, bulimia, anorexia o trastorno bipolar (en estos últimos se puede precipitar un episodio maniaco). También está contraindicado usarlo junto a inhibidores de la MAO, ya que la toxicidad aguda de bupropion se potencia con estos fármacos, produciendo agitación, cambios psicóticos y convulsiones ${ }^{30}$.

No se recomienda administrarlo en el embarazo ni lactancia, ya que puede atravesar la barrera placentaria y se puede excretar en la leche materna.

\section{Vareniclina}

Vareniclina es un agonista parcial de los receptores nicotínicos de acetilcolina $\alpha 4 \beta 2$, recientemente aprobado por la FDA para el tratamiento del tabaquismo. Está disponible en Chile desde Abril de 2007 y se ha convertido en una alternativa atractiva, no sólo por su novedoso mecanismo de acción, sino también por su alta eficacia y buena tolerancia reportada en varios estudios.

\section{Propiedades farmacológicas:}

La dependencia a nicotina se debe en parte a su actividad agonista en los receptores nicotínicos $\alpha 4 \beta 2$. Al ser estimulados, se produce liberación de dopamina en el núcleo accumbens, neurotransmisor que produce los efectos placenteros buscados por el fumador. Cuando éste 
deja de fumar, la ausencia de nicotina disminuye los niveles de dopamina, produciendo urgencia por fumar ("craving"), un importante contribuyente para las recaídas.

Diversos estudios sugieren que al estimular estos receptores con un agonista parcial como la vareniclina, aumentarían los niveles de dopamina, aliviando los síntomas de privación. Además, la unión competitiva con los receptores $\alpha 4 \beta 2$, bloquearía al menos parcialmente la activación dopaminérgica provocada por la nicotina en caso de recaída. Este doble mecanismo sería el responsable de la eficacia de la vareniclina como tratamiento del tabaquismo ${ }^{34}$.

Se ha observado además que este nuevo medicamento, al no ser metabolizado en el citocromo $\mathrm{P} 450$, no altera la farmacocinética de varios medicamentos (TRN, bupropión, warfarina, digoxina, cimetidina, y metformina) convirtiéndolo en un medicamento seguro ${ }^{35}$.

\section{Eficacia terapéutica}

- Tasa de abstinencia

La eficacia de la terapia de 12 semanas con vareniclina $1 \mathrm{mg} 2$ veces al día para el tratamiento del tabaquismo, fue comparada con bupropion $150 \mathrm{mg} 2$ veces al día y placebo en 2 estudios randomizados, multicéntricos, en fase III $^{36,37}$

En ambos estudios, a las 12 semanas aquellos participantes del grupo de vareniclina lograron tasas de abstinencia continua significativamente mayor que en los otros grupos. Los resultados fueron similares en ambos trabajos con un OR de 1,9 para vareniclina versus bupropion $(\mathrm{p}<0,001)$ y un OR de 3,9 para vareniclina versus placebo $(\mathrm{p}<0,001)$.

A largo plazo (semana 52), la tasa de abstinencia fue significativamente mayor con vareniclina que con bupropion en uno de los estudios (OR 1,8; IC 95\% 1,2-2,6; $\mathrm{p}=0,004$ ), pero no en el otro (OR 1,5; IC 95\% 1,0-2,2, p = 0,05). En ambos estudios vareniclina fue superior a placebo (OR 3,1 y OR 2,7, ambos con $\mathrm{p}<0,001$ ).

\section{- Mantención de abstinencia}

Un estudio multicéntrico ${ }^{38}$ incluyó 1.210 fumadores que recibieron vareniclina por 12 semanas y que lograron abstinencia durante este período. Luego fueron randomizados en 2 grupos; en uno se siguió usando vareniclina y en el otro placebo, durante 12 semanas más. La tasa de abstinencia a las 52 semanas, fue mayor en el grupo que recibió vareniclina por 24 semanas, con un OR de 1,34 (IC 95\% 1,06-1,69, $\mathrm{p}=0,02)$.

\section{- Otros efectos}

Vareniclina disminuye los deseos de fumar en forma significativamente mayor que placebo, aunque su efecto en síntomas de privación es menos consistente. Vareniclina tiene otros efectos al compararlo con placebo: disminuye el cansancio, el insomnio y los efectos reforzantes del tabaco.

\section{Efectos secundarios:}

El efecto secundario más frecuente son las náuseas, alcanzando un 34,9\% en la mayoría de los casos, son leves a moderadas y disminuyen en el tiempo. La discontinuación de vareniclina por efectos adversos es $10,5 \%$, comparado con $12,6 \%$ y $7,3 \%$ en los grupos de bupropion y placebo, respectivamente $^{37}$.

Además se ha observado un aumento de peso de 2,89 $\mathrm{kg}$ en promedio, versus un 1,8 y $3,1 \mathrm{~kg}$ en los grupos de bupropion y placebo respectivamente ${ }^{39}$. Estos resultados sugieren que el aumento de peso es consecuencia de dejar de fumar, más que este relacionado con vareniclina perse.

\section{Recomendaciones de uso}

La dosis recomendada es $1 \mathrm{mg}$ dos veces al día, después de las comidas. El tratamiento debiera empezar una semana antes de la fecha programada para dejar de fumar. La vareniclina debiera ser titulada durante una semana, usando dosis progresivamente mayores hasta lograr la dosis objetivo. Con este esquema, la incidencia de náuseas disminuye significativamente ${ }^{34}$.

La duración recomendada es de 12 semanas, y para aquellos que logran abstinencia durante ese período podrían recibir vareniclina por otras 12 semanas extras, lo que aumentaría las probabilidades de mantener la abstinencia a largo plazo.

A modo de resumen, en la Tabla 3 se presentan los medicamentos aprobados por la FDA.

\section{Otras drogas para el tratamiento del tabaquismo}

\section{Clonidina}

Es un agonista $\alpha 2$ noradrenérgico. En un meta análisis reciente de 6 ensayos clínicos randomizados, comparando clonidina y placebo se observaron mayores tasas de cese de tabaco en el primer grupo. Sólo uno de los estudios mostró valores estadísticamente significativos, cuyo OR es comparable con cualquier TRN (OR 1,89, IC 95\%: $1,3-2,7)^{40}$. 
Tabla 3. Características de los principales medicamentos para el tratamiento del tabaquismo, aprobados por la FDA

\begin{tabular}{|c|c|c|c|c|}
\hline Droga & Mecanismo de acción & OR (IC 95\%) & Ventajas & Desventajas \\
\hline TRN & $\begin{array}{l}\text { Reducción de síntomas } \\
\text { de privación y de efec- } \\
\text { tos reforzantes; pro- } \\
\text { ducción de efectos bus- } \\
\text { cados previamente en } \\
\text { los cigarrillos }\end{array}$ & & & Mareos, náuseas \\
\hline Chicle & & $1,7(1,5-1,8)$ & $\begin{array}{l}\text { Simula ritual oral, } \\
\text { uso SOS }\end{array}$ & $\begin{array}{l}\text { Dispepsia, dolor } \\
\text { mandibular }\end{array}$ \\
\hline Parche & & $1,8(1,6-2,0)$ & $\begin{array}{l}\text { Mejor adherencia, } \\
\text { niveles estables de } \\
\text { nicotina }\end{array}$ & $\begin{array}{l}\text { Irritación local, } \\
\text { trastornos del sueño }\end{array}$ \\
\hline Inhalador & & $2,1(1,4-3,2)$ & $\begin{array}{l}\text { Simula ritual oral, } \\
\text { uso SOS }\end{array}$ & $\begin{array}{l}\text { Irritación de boca y } \\
\text { faringe }\end{array}$ \\
\hline Spray nasal & & $2,4(1,6-3,4)$ & $\begin{array}{l}\text { Simula ritual oral, } \\
\text { uso SOS }\end{array}$ & $\begin{array}{l}\text { Irritación local, } \\
\text { rinorrea }\end{array}$ \\
\hline \multirow[t]{2}{*}{ Bupropion } & $\begin{array}{l}\text { Mediado por mecanismos } \\
\text { noradrenérgicos y/o }\end{array}$ & & & \\
\hline & dopaminérgicos & $2,1(1,8-2,4)$ & $\begin{array}{l}\text { Escasos efectos } \\
\text { adversos, previene } \\
\text { aumento de peso }\end{array}$ & Insomnio, boca seca \\
\hline Vareniclina & $\begin{array}{l}\text { Agonista parcial de los } \\
\text { receptores nicotínicos } \\
\alpha 4 \beta 2 \text {; además bloquea la } \\
\text { acción de la nicotina }\end{array}$ & $\begin{array}{l}3,1(2,0-4,9) y \\
2,7(1,7-4,1)\end{array}$ & $\begin{array}{l}\text { Reduce síndrome de } \\
\text { privación y efectos } \\
\text { reforzantes del tabaco }\end{array}$ & Náuseas \\
\hline
\end{tabular}

FDA: Food and Drug Administration de EE.UU. TRN: Terapia de reemplazo de nicotina. Modificado de Keating ${ }^{34}$

Los efectos adversos más observados son sequedad de boca (25-40\%), sedación (12-35\%), hipotensión $(15 \%)$ y constipación $(10 \%)^{41}$.

En general, la clonidina no es tan efectiva como la TRN en el tratamiento del tabaquismo y su alta tasa de efectos adversos limita su uso.

\section{Nortriptilina}

Antidepresivo tricíclico que bloquea la recaptura de noradrenalina y serotonina, mejorando así síntomas de privación y síntomas depresivos post-cesación. Distintos estudios han demostrado que es dos veces más efectivo que placebo $(\mathrm{OR}=2,79 \text {, IC 95\%: } 1,7-4,5)^{42}$. Sin embargo, un estudio comparativo reciente de nortriptilina y bupropion mostró superioridad significativa de bupropion (42 versus $31 \%$ de abstinencia a los 6 meses ${ }^{43}$. Los efectos secundarios más frecuentes son sequedad de boca y constipación, que se presentan en alto número de pacientes ${ }^{44}$.

Con los avances en el conocimiento de la neurofarmacología de la nicotina en los últimos años se están desarrollando y estudiando nuevos medicamentos de efectividad variable, algunos de los cuales se muestran en la Tabla 4.
Para finalizar se revisará la vacuna antinicotina y el rimonabant, cuyos estudios en desarrollo, podrían aportar novedades en los próximos años.

\section{Vacuna antinicotina}

La vacuna antinicotina induce producción de anticuerpos anti-moléculas de nicotina, evitando el cruce de ésta por la barrera hematoencefálica y disminuyendo su tasa de metabolismo. Estudios en animales han mostrado una reducción del $65 \%$ de la nicotina que alcanza los receptores nicotínicos cerebrales y una disminución significativa de la cantidad de dopamina producida por la estimulación por nicotina ${ }^{45}$.

Existen tres compañías que están desarrollando las vacunas (Xenova, Nabi y Cytos), cuyos estudios en fase 2 no han concluido, por lo que la evidencia es limitada. Los resultados de estudios en fase 1 sugieren que estas vacunas son seguras, bien toleradas e inmunogénicas ${ }^{46}$.

El estudio de Cytos reportó que la eficacia de la vacuna dependía de la respuesta inmunogénica del fumador; en el grupo de vacuna versus placebo no se observaron diferencias significativas, pero al realizar un estudio por subgrupo se encontró una alta tasa de éxito en los pacientes 
Tabla 4. Otros medicamentos en estudio para el tratamiento del tabaquismo

\begin{tabular}{|c|c|c|c|}
\hline Droga & Eficacia & Ventajas & Desventajas \\
\hline $\begin{array}{l}\text { I-MAO } \\
\text { Lazabemide } \\
\text { Selegilina } \\
\text { Moclobemida }\end{array}$ & $\begin{array}{l}\text { Pocos estudios, escasa } \\
\text { eficacia }\end{array}$ & Bien tolerados & $\begin{array}{l}\text { Toxicidad hepática e } \\
\text { hipertensión arterial }\end{array}$ \\
\hline ISRS & $\begin{array}{l}6 \text { ensayos clínicos (mayoría } \\
\text { con fluoxetina); no conclu- } \\
\text { yentes }\end{array}$ & $\begin{array}{l}\text { Efectivo en ciertos grupos } \\
\text { (deprimidos), previene } \\
\text { aumento de peso }\end{array}$ & $\begin{array}{l}\text { Trastornos del sueño, } \\
\text { disminución líbido, náuseas }\end{array}$ \\
\hline Fluoxetina & & $\begin{array}{l}\text { Mayor tasa de abstinencia } \\
\text { en gente con altos niveles } \\
\text { de síntomas depresivos }\end{array}$ & \\
\hline Venlafaxina & & $\begin{array}{l}\text { Más efectivo que placebo } \\
\text { sólo en fumadores "livianos" }\end{array}$ & \\
\hline Naltrexona & $\begin{array}{l}\text { Estudios pequeños y resul- } \\
\text { tados inconsistentes }\end{array}$ & $\begin{array}{l}\text { Podría limitar ganancia de } \\
\text { peso y tratar policonsumo }\end{array}$ & $\begin{array}{l}\text { Altos efectos adversos } \\
\text { (náuseas, sedación) }\end{array}$ \\
\hline Gabapentina & Un estudio pequeño & $\begin{array}{l}\text { Podría bloquear síndrome } \\
\text { de privación y recompensa }\end{array}$ & $\begin{array}{l}\text { Seguridad a largo plazo } \\
\text { desconocida }\end{array}$ \\
\hline Mecamilamina & $\begin{array}{l}\text { Dos estudios pequeños, } \\
\text { ambos asociados a parche } \\
\text { de nicotina }\end{array}$ & Buena tolerancia & Eficacia sólo asociada a TRN \\
\hline Topirimato & $\begin{array}{l}\text { Un estudio con fumadores } \\
\text { alcohólicos }\end{array}$ & $\begin{array}{l}\text { Efectivo en ciertos subgrupos } \\
\text { (alcohólicos fumadores) }\end{array}$ & $\begin{array}{l}\text { Sólo estudiado en dependien- } \\
\text { tes de alcohol }\end{array}$ \\
\hline
\end{tabular}

I-MAO: Inhibidores de la monoaminoxidasa; ISRS: Inhibidores selectivos de la recaptación de serotonina; modificado de Schnoll \& Lerman ${ }^{51}$

con respuesta inmunogénica elevada (57 versus $31 \%)^{47}$.

En el estudio de Nabi, se observó una tasa de éxito del $40 \%$ en aquellos que recibieron dosis de $200 \mu \mathrm{g}$, comparado con una tasa de éxito $<10 \%$ en grupos con menores dosis o placebo ${ }^{48}$.

$\mathrm{Si}$ bien estos resultados son alentadores, la evidencia actual es limitada y sacar conclusiones sobre la seguridad y eficacia es aún prematuro.

\section{Rimonobant}

Los receptores canabinoides 1 (CB1) modulan la liberación de dopamina. Según modelos animales, un antagonista del receptor CB1, rimonobant, reduce la auto-administración de nicotina y aumenta las concentraciones de serotonina, dopamina y noradrenalina a nivel cortical y en el núcleo accumbens ${ }^{49}$. Así, el rimonabant podría no sólo atenuar las propiedades reforzantes de la nicotina, sino también reducir las recaídas.

Clínicamente, se han observado diferencias significativas pero discretas de rimonabant 20 $\mathrm{mg}$ al día usado durante 10 semanas versus placebo (27,6 versus $16,1 \%$ de abstinencia a las 42 semanas de seguimiento $)^{50}$. Además, con el uso de rimonabant como terapia de mantención, el aumento de peso es significativamente menor que con placebo al cabo de 1 año ${ }^{51}$.

Este fármaco representaría una alternativa atractiva para aquellos fumadores obesos en quienes el aumento de peso al dejar de fumar es un problema mayor, pero se requieren más estudios para demostrar su real eficacia.

Una línea de trabajo promisoria a largo plazo son los estudios farmacogenéticos de las drogas, que permitirían predecir el efecto de los medicamentos en determinados subgrupos de fumadores caracterizados de acuerdo a estudios genéticos específicos. Así, medicamentos que parecieran no ser útiles en el conjunto de pacientes, sí podrían ser beneficiosos en un subgrupo de éstos ${ }^{52}$. A modo de ejemplo, recientemente se publicó un estudio ${ }^{53}$ cuyo objetivo fue evaluar la influencia genética en la respuesta al tratamiento del tabaquismo con bupropion: los resultados sugieren que los diferentes polimorfismos del receptor de dopamina pueden influir en la respuesta al tratamiento con bupropion. Nuevos estudios, con poblaciones mayores, permitirán continuar avanzando en esta interesante línea de trabajo. 


\section{Comentarios finales}

Después de analizar las alternativas terapéuticas existentes hoy en día y de revisar lo que puede venir en los próximos años, al comprenderse mejor los mecanismos psico-farmacológicos de la adicción al tabaco, no podemos sino estar muy optimistas en que hoy se pueden ofrecer más y mejores terapias que hace algunos años. Los médicos tenemos la obligación de ofrecer apoyo y tratamiento a todo fumador que manifieste intención de dejar de fumar. Por otra parte, los pacientes pueden y deben solicitar dicha ayuda, habiendo hoy mejores expectativas de éxito para el abandono definitivo de consumo de tabaco. De esta manera se estará contribuyendo a disminuir en forma significativa las tasas de mortalidad producidas por el tabaquismo en nuestro país y en el mundo.

\section{Bibliografía}

1.- MINISTERIO DE SALUD. Departamento de Estadísticas e Información en Salud. 2006. http://deis.minsal. $\mathrm{cl} /$ deis/tabaco/muertes.htm

2.- MINISTERIO DE SALUD. Departamento de Epidemiología y Departamento de Promoción de la Salud. Encuesta Nacional de Calidad de Vida. Octubre, 2001.

3.- MINISTERIO DE SALUD. Departamento de Epidemiología. Encuesta Nacional de Salud. Mayo, 2004. http://epi.minsal.cl/epi/html/elvigia/vigia15.pdf

4.- CONSEJO NACIONAL PARA EL CONTROL DE ESTUPEFACIENTES (CONACE). Ministerio del Interior. Gobierno de Chile. Sexto Estudio Nacional de Drogas en Población General de Chile. 1994-2004.

5.- CONSEJO NACIONAL PARA EL CONTROL DE ESTUPEFACIENTES (CONACE). Ministerio del Interior. Gobierno de Chile. Sexto Estudio Nacional de Drogas en Población Escolar de Chile 2005. Santiago de Chile. Julio de 2006.

6.- Primer informe Encuesta Mundial sobre tabaquismo en Jóvenes (EMTA JOVEN). Santiago de Chile. 2001. Universidad de Chile. Facultad de Medicina. Escuela de Salud Pública http://www.cdc.gov/tobacco/Global/GYTS/ reports/paho/2001/chile2001_sp.htm

7.- MINISTERIO DE SALUD. Objetivos Sanitarios y Modelo de Salud para la década 2000-2010. Enero, 2002.

8.- US DEPARTMENT OF HEALTH AND HUMAN SERVICES. The health consequences of smoking: A report of the Surgeon General, 2004. www.cdc.gov/ tobacco/sgr/sgr_2004/index.htm

9.- EZZATI M, LÓPEZ A D. Estimates of global mortality attributable to smoking in 2000. Lancet 2003; 362: 847-52.

10.- DOLL R, PETO R, BOREHAM J, SUTHERLAND I. Mortality in relation to smoking: 50 years observations on male British doctors. BMJ 2004; 328: 1529-33.

11.- US DEPARTMENT OF HEALTH AND HUMAN SERVICES. Nicotine addiction: A report of the Surgeon General. Rockville, US Dept. of Health and Human
Services, 1988.

12.- AMERICAN PSYCHIATRIC ASSOCIATION. Diagnostic and statistical manual of mental disorders. 4th ed., text revision: DSM-IV-TR. Washington, D.C.: American Psychiatric Association; 2000.

13.- WORLD HEALTH ORGANIZATION. International Classification of Diseases, 10th Edn. Geneva, World Health Organization, 1992.

14.- PONCIANO G. Bases científicas de la vareniclina. Guía para la práctica clínica: Sistema para la cesación del tabaquismo. American College of Chest Physicians, 2006.

15.- FOULDS J. The neurobiological basis for partial agonist treatment of nicotine dependence: varenicline. Int J Clin Pract, May 2006; 60: 5: 571-6.

16.- GRANDA ORIVE J I. El tabaquismo como enfermedad adictiva crónica. En: Jiménez-Ruiz CA, Fagerström KO (eds). Tratado de Tabaquismo, 2a edición. Madrid. Ergon 2007; 99-119.

17.- GUIDELINE PANEL, STAFF AND CONSORTIUM REPRESENTATIVES. The tobacco use and dependence clinical practice guideline. A clinical practice guideline for treating tobacco use and dependence. US Public Health Service Report. JAMA 2000; 283: 324454.

18.- HENNINGFIELD J E. Nicotine medications for smoking cessation. N Engl Med, 1995; 333: 1196-203.

19.- BARTOLOMÉ MORENO B, PÉREZ TRULLÉN A, CLEMENTE JIMÉNEZ M L. Tratamiento farmacológico del tabaquismo: terapia sustitutiva con nicotina. En: Jiménez-Ruiz CA, Fagerström KO (eds). Tratado de Tabaquismo, 2a edición. Madrid. Ergon 2007; 355-67.

20.- FIORE MC, BAILEY WC, COHEN SJ et al. Treating Tobacco Use and Dependence. Clinical Practice Guideline. Rockville, MD: U.S. Department of Health and Human Services, Public Health Service; 2000.

21.- RUSSELL M A H, RAW M, JARVIS M J. Clinical use of nicotine chewing gum. BMJ 1980; 280: 1599-602.

22.- HURT R D. Treating Tobacco Dependence in a Medical Setting: Best Practices. Nicotine Dependence Center, The Mayo Clinic, 2006.

23.- SHIFFMAN S, ELASH C A, PATON S M, et al. Comparative efficacy of 24-hour and 16-hour transdermal nicotine patches for relief of morning craving. Addiction 2000; 96: 1185-95.

24.- SCHNEIDER N G, LUNELL E, OLMSTEAD R E, FAGERSTROM K O. Clinical pharmacokinetics of nasal nicotine delivery. A review and comparison to other nicotine systems. Clin Pharmacokinet 1996; 31: 6580.

25.- NIAURA R, SAYETTE M A, SHIFFMAN S, et al. Comparative efficacy of rapid-release nicotine gum $v s$ Nicorette in relieving smoking cue-provoked craving. (Resumen). Annual Meeting of the Society for Research on Nicotine and Tobacco: February 19-22, 2003; New Orleans, LA.

26.- FAGERSTROM K O, SCHNEIDER N G, LUNELL E. Effectiveness of nicotine patch and nicotine gum as individual versus combined treatments for tobacco withdrawal symptoms. Psychopharmacology 1993; 111: 271-7.

27.- KORNITZER M, BOUTSEN M, DRAMAIX M, THIJS J, GUSTAVSSON G. Combined use of nicotine patch and gum in smoking cessation: a placebocontrolled clinical trial. Prev Med 1995; 24: 41-7.

28.- FAGERSTROM K O, HUGHES J R. Nicotine concentrations with concurrent use of cigarretes and 
nicotine replacement: a review. Nicotine Tob Res 2002; 4 (Suppl 2): S73-9.

29.- CARRERAS J M. Tratamiento farmacológico no nicotínico del tabaquismo. En: Calvo JR, López A (eds). El Tabaquismo. Madrid. Cometa. 2003.

30.- RAMOS PINEDO A, OTEEN B. Tratamiento farmacológico del tabaquismo: bupropion. En: JiménezRuiz CA, Fagerström KO (eds). Tratado de Tabaquismo, $2^{a}$ edición. Madrid. Ergon 2007; 369-79.

31.- WU P, WILSON K, DIMOULAS P, MILLS E J. Efectiveness of smoking cessation therapies: a systematic review and meta-analysis. BMC Public Health 2006; 6: 300.

32.- HENNINGFIELD J E, FANT R V, BUCHHALTER A R, STITZER M L. Pharmacotherapy for Nicotine Dependence. CA Cancer J Clin 2005; 55: 281-99.

33.- HUGHES J, STEAD L, LANCASTER T. Antidepressants for smoking cessation. Cochrane Database Syst Rev 2007: CD000031.

34.- KEATING G M, SIDDIQUI M A A. Varenicline: a review of its use as and aid to smoking cessation therapy. CNS Drugs 2006; 20: 945-60.

35.- POTTS LA, GARWOOD C L. Varenicline: the newest agent for smoking cessation. Am J Health Syst Pharm. 2007; 64: 1381-4.

36.- GONZÁLES D, RENNARD S I, NIDES M, ONCKEN C, AZOULAY S, BILLING C B, et al. Varenicline, an $\alpha 4 \beta 2$ nicotinic acetylcholine receptor partial agonist, vs sustained release bupropion and placebo for smoking cessation: an randomized controlled trial. JAMA 2006; 296: 47-55.

37.- JORENBY D E, HAYS J T, RIGOTTI N A, AZOULAY S, WATSKY E J, WILLIAMS K E, et al. Efficacy of varenicline, an $\alpha 4 \alpha \beta 2$ nicotinic acetylcholine receptor partial agonist, vs placebo or sustained-release bupropion for smoking cessation:a randomized controlled trial. JAMA 2006; 296: 56-63.

38.- TONSTAD S, TØNNESEN P, HAJEK P, WILLIAMS $\mathrm{K}$ E, BILLING C B, REEVES K R, et al. Effect of maintenance therapy with varenicline on smoking cessation: a randomized controlled trial. JAMA 2006; 296: 64-71.

39.- ONCKEN C, GONZÁLES D, NIDES M, RENNARD S, WATSKY E, BILLING C B, et al. Efficacy and safety of the novel selective nicotinic acetylcholine receptor partial agonist, varenicline, for smoking cessation. Arch Intern Med 2006; 166: 1571-7.

40.- GOURLAY S G. Clonidine for smoking cessation (Review). The Cochrane Collaboration. Wiley Publishers (2006): 1-10.

41.- GOURLAY S G, BENOWITZ N L. Is clonidine an effective smoking cessation therapy? Drugs 1995; 50:
197-207.

42.- PROCHAZKA A V, WEAVER M J, KELLER R T, FRYER G E, LICARI P A, LOFASO D. A randomized trial of nortryptiline for smoking cessation. Arch Intern Med 1998; 158: 2035-9.

43.- HAGGSTRÄM F M, CHATKIN J M, SUSSENBACHVAZ E, CESARI D H, FAM C F, FRITSCHER C C. A controlled trial of nortriptyline, sustained-release bupropion and placebo for smoking cessation: preliminary results. Pulm Pharmacol Ther 2006; 19: 205-9.

44.- WAGENA E J, KNIPSCHILD P, ZEEGERS M P. Should nortriptyline be used as a first-line aid to help smokers quit? Results from a systematic review and meta-analysis. Addiction 2005; 100: 317-26.

45.- MAURER P, JENNINGS G T, WILLERS J, ROHNER F, LINDMAN Y, ROUBICEK K, et al. A therapeutic vaccine for nicotine dependence: preclinical efficacy, and Phase I safety and immunogenicity. Eur J Inmmunol 2005; 35: 2031-40.

46.- DE GRANDA ORIVE J I. When will the nicotine vaccine be ready? Arch Bronconeumol 2005; 41: 2-4.

47.- CORNUZ J, KILINGLER K, MUELLER P. A therapeutic vaccine for nicotine dependence: results of Phase I and a randomized Phase II study. Presented at: The American Society of Clinical Oncology. Orlando, Florida, USA; 2005.

48.- HATSUKAMI D K, RENNARD S, JORENBY D, FIORE M, KOOPMEINERS J, DE VOS A, et al. Safety and immunogenicity of a nicotine conjugate vaccine in current smokers. Clin Pharmacol Ther 2005; 78: 456-67.

49.- COHEN C, KODAS E, GRIEBEL G. CB1 receptor antagonists for the treatment of nicotine addiction. Pharmacol Biochem Behav 2005; 81: 387-95.

50.- DALE L, ANTHENELLI R. Rimonabant as an aid to smoking cessation in smokers motivated to quit: results from a US multicenter study-STRATUS-US trial. American College of Cardiology, New Orleáns, USA, 2004.

51.- CAHILL K, USSHER M. Cannabinoid type 1 receptor antagonists (rimonabant) for smoking cessation. Cochrane Database Syst Rev 2007 Jul 18; (3): CD005353.

52.- SCHNOLL R A, LERMAN C. Current and emerging pharmacotherapies for treating tobacco dependence. Expert Opin. Emerging Drugs 2006; 11 (3).

53.- DAVID S P, BROWN R A, PAPANDONATOS G D, KAHLER C W, LLOYD-RICHARDSON E E, MUNAFÓ M R, et al. Pharmacogenetic clinical trial of sustained-release bupropion for smoking cessation. Nicotine \& Tobacco Research 2007; 9, 8: 821-33.

Correspondencia a:

Dr. Sergio Bello S.

Instituto Nacional del Tórax

JM Infante 717, Providencia

Santiago

E-mail: sbello@torax.cl 